\title{
Phase separation of binary mixtures in shear flow: A numerical study
}

\author{
F. Corberi \\ Istituto Nazionale per la Fisica della Materia, Unità di Salerno and Dipartimento di Fisica, \\ Università di Salerno, 84081 Baronissi (Salerno), Italy \\ G. Gonnella \\ Istituto Nazionale per la Fisica della Materia, Unità di Bari and Dipartimento di Fisica, Università di Bari, \\ and Istituto Nazionale di Fisica Nucleare, Sezione di Bari, Via Amendola 173, 70126 Bari, Italy
}

A. Lamura

Institut für Festkörperforschung, Forschungszentrum Jülich, 52425 Jülich, Germany

(Received 27 July 2000)

\begin{abstract}
The phase-separation kinetics of binary fluids in shear flow is studied numerically in the framework of the continuum convection-diffusion equation based on a Ginzburg-Landau free energy. Simulations are carried out for different temperatures both in $d=2$ and 3. Our results confirm the qualitative picture put forward by the large- $N$ limit equations studied by Corberi et al. [Phys. Rev. Lett. 81, 3852 (1998)]. In particular, the structure factor is characterized by the presence of four peaks whose relative oscillations give rise to a periodic modulation of the behavior of the rheological indicators and of the average domains sizes. This peculiar pattern of the structure factor corresponds to the presence of domains with two characteristic thicknesses, whose relative abundance changes with time.
\end{abstract}

PACS number(s): 47.20.Hw, 05.70.Ln, 83.50.Ax

\section{INTRODUCTION}

Binary fluids quenched below the demixing temperature exhibit an interesting off-equilibrium phenomenology that is nowadays reasonably well understood [2]. Typically, after an early stage when domains of the two phases are formed, the kinetics proceeds by a coarsening of these structures while keeping their morphology invariant. For viscous fluids, such as some polymer blends, hydrodynamic effects can be neglected in a preasymptotic time domain, and a description of the dynamics in terms of diffusive processes alone is appropriate. This is the situation we focus on in this paper. In this case, the typical domain size $R(t)$ grows according to the Lifshitz-Slyozov law $R(t) \sim t^{\alpha} \sim t^{1 / 3}$ [3]. This whole phenomenology is known to be correctly described in terms of the time dependent Ginzburg-Landau model [2]. This continuum approach, beside being often more efficient for numerical investigations, is better suited for analytical purposes. In fact, despite the fact that an exact solution is presently lacking, successful approximate theories have been developed. Among these, a prominent role is played by the large- $N$ expansion. Indeed, when the model is generalized to a vectorial order parameter with an arbitrary number $N$ of components, the limit $N \rightarrow \infty$ turns out to be analytically tractable, and perturbation theories around it can be applied. This special limit has proven to provide qualitatively correct information about the kinetic evolution, contributing, in some cases, to clarifying the nature of the process [4].

When segregating systems are driven mechanically, by applying an external field or making the liquid flow, the comprehension we have of the kinetics is much poorer. This is at odds with the wide technological interest in these systems in many application areas [5]. In the case of applied shear flow considered here, it is known that the evolution is profoundly changed with respect to that of a static liquid [6].
The most noticeable effect is the alignment of the domains of the two species along the flow direction, chosen in the following as the $x$ axis. This produces an anisotropy in the system and, in principle, one has to consider the typical domains sizes $R_{x}, R_{y}$, and $R_{z}$ along the coordinate axis separately. Modified growth exponents for $R_{x}, R_{y}$, and $R_{z}$ with respect to the case of an immobile fluid are expected, and a difference $\alpha_{x}-\alpha_{y}=1$ between the power-law exponents of $R_{x}$ and $R_{y}$ was reported [7,8]. In experiments, depending on the system considered, it was also observed that, after an initial growth, the shear can stabilize the system into a stationary state characterized by a very large $R_{x}$, with $R_{y}$ finite; that is, the phase separation process is interrupted [9-12].

Stretching of domains requires work against surface tension. Macroscopically, the viscoelastic response of the fluid shows up through a nonvanishing stress tensor $[13,14]$. On microscopic scales it was recognized [15] that the strain exerted by the fluid can produce different effects, in particular the breakup and recombination of domains. The fragmentation process is accompanied by the formation of small bubbles and more isotropic clusters. Then these structures, which grow by diffusion and join each other favored by the flow, are successively stretched again and broken in a cyclical way. In an extended system, with many such domains, if the breakup events occur incoherently along the fluid we do not expect any appreciable effect, possibly apart from a global slowing of the growth process. Differently, if the breakup of the domains with successive, more isotropic, growth is a coherent process in the network, occurring when the stress exceeds a sort of threshold reached syncronously in all the system, one could expect a periodicity superimposed on the net behavior of the typical domains size and of other observables.

In a previous paper [1] we showed that a pattern similar to the latter case outlined above is indeed exhibited by the 
large- $N$ model: Physical observables such as the domain radii or the rheological indicators behave for long times as power laws decorated by log-time periodic oscillations. In this framework it was possible to identify this oscillatory pattern with a corresponding behavior of the four peaks observed in the structure factor. Moreover, in the large- $N$ limit it was possible to show the existence of a dynamical scaling regime even in the presence of shear with the difference between the power-law growth exponents in the flow and in the transverse directions equal to 1 . This last observation was confirmed by a successive exact asymptotic solution of the large- $N$ model [16]. However, in the $t \rightarrow \infty$ limit, the only accessible in this solution, the periodic oscillations do not survive. Then it must be believed that these are long lasting preasymptotic effects. In experiments, on the other hand, a four-peaked structure factor was occasionally reported [17], and the so called double overshoot of the excess viscosity observed sometimes [18] can be interpreted as being due to the oscillations of this quantity.

Given this scenario, the question of the accuracy of the large- $N$ limit for the description of real systems, where $N$ $=1$, must be addressed. In fact, the absence of topological defects for $N>d$ makes concepts like domains or interfaces inappropriate, and the reliability of the approximation, even qualitatively, is not for granted. Concerning this issue important topics to be addressed are, in our opinion, (i) the existence of the oscillatory pattern together with its counterpart of a fourfold peaked structure factor and (ii) the existence of a scaling regime with specific growth exponents. We do so in this paper by a complete numerical investigation of a timedependent Ginzburg-Landau model which can be considered as a preliminary step before the study of a fully hydrodynamic model. A preliminary account of our results at $T=0$ in $d=2$ was already published in a previous paper [19]. There, the existence of structure factors with four peaks was confirmed and interpreted in terms of domains distributed in the system with two typical thicknesses. Here we overview and detail these results further. Moreover, we study the effects of thermal fluctuations and the role of spatial dimensionality by comparing results for two- and three-dimensional systems.

Regarding issue (i), our results are confirmative of the large- $N$ prevision: four peaks of the structure factor are resolved, and an initial oscillation of the typical domain radii and other quantities is observed. Domains with different thickness and structure factors with four peaks are also observed in quenching at finite temperatures and in threedimensional systems. With respect to point (ii), despite the fact that $R_{x}$ keeps growing, CPU limitations do not allow a determination of the the behavior of $R_{y}$ and $R_{z}$ on sufficiently long time scales to detect a possible power-law behavior and the actual value of the exponents. Our data only provide a rough semiquantitative agreement with the expected scaling laws which, therefore, cannot be inferred presently from numerical simulations alone.

This paper is organized as follows. In Sec. II we introduce the time-dependent Ginzburg-Landau model used in the simulations. In Secs. III and IV, we present the results of the numerical simulations in $d=2$ and 3, respectively. Finally we summarize, discuss some open problems and draw our conclusions.

\section{MODEL}

We consider the Ginzburg-Landau free energy

$$
\mathcal{F}\{\varphi\}=\int d^{d} x\left\{\frac{a}{2} \varphi^{2}+\frac{b}{4} \varphi^{4}+\frac{\kappa}{2}|\nabla \varphi|^{2}\right\},
$$

where $\varphi$ is the order parameter representing the concentration difference between the two components. The polynomial terms in the free-energy density have a single-well structure when $a>0, b>0$, and describe the disordered state of the mixture with $\langle\varphi=0\rangle$. In the ordered state, for $a<0$ and $b>0$, two symmetric minima are located at $\varphi$ $=\sqrt{-a / b}$. These are the equilibrium values of the order parameter in the small temperature limit. The gradient-squared term in Eq. (1), with $\kappa>0$, takes into account the energy cost of interfaces between domains of different composition. The kinetics is described by the convection-diffusion equation

$$
\frac{\partial \varphi}{\partial t}+\vec{\nabla} \cdot(\varphi \vec{v})=\Gamma \nabla^{2} \frac{\delta \mathcal{F}}{\delta \varphi}+\eta
$$

where the order parameter is coupled to an external velocity field $\vec{v}$. Here $\Gamma$ is a mobility coefficient, and $\vec{v}$ will be assumed to be a plane shear flow profile, namely,

$$
\vec{v}=\gamma y \vec{e}_{x}
$$

$\gamma$ and $\vec{e}_{x}$ being a spatially uniform shear rate [6] and a unit vector in the $x$ direction, respectively. The thermal noise $\eta$, which describes thermal fluctuations [6], has zero mean, and satisfies the fluctuation-dissipation relationship

$$
\left\langle\eta(\vec{r}, t) \eta\left(\vec{r}^{\prime}, t^{\prime}\right)\right\rangle=-2 T \Gamma \nabla^{2} \delta\left(\vec{r}-\vec{r}^{\prime}\right) \delta\left(t-t^{\prime}\right),
$$

where $T$ is the temperature of the fluid, and $\langle\cdots\rangle$ denotes the ensemble average. The validity of the present approach is restricted to systems where hydrodynamic effects can be neglected. When hydrodynamics comes into play, a model that also takes into account the contribution coming from the Navier-Stokes equation should be used [20]. For weakly sheared polymer blends with a large polymerization index and similar mechanical properties of the two species, however, the present model is expected to be satisfactory in a preasymptotic time domain when velocity fluctuations are small [2].

Equation (2) can be cast in a dimensionless form after a redefinition of time, space and field scales [21]. Then we have chosen $\Gamma=|a|=b=\kappa=1$.

The main observable for the study of the growth kinetics is the structure factor $C(\vec{k}, t)=\langle\varphi(\vec{k}, t) \varphi(-\vec{k}, t)\rangle$, namely, the Fourier transform of the real-space equal time correlation function. From the knowledge of the structure factor one computes the average size of domains in the different directions. These quantities can be defined as

$$
R_{x}(t)=\pi \frac{\int d \vec{k} C(\vec{k}, t)}{\int d \vec{k}\left|k_{x}\right| C(\vec{k}, t)},
$$



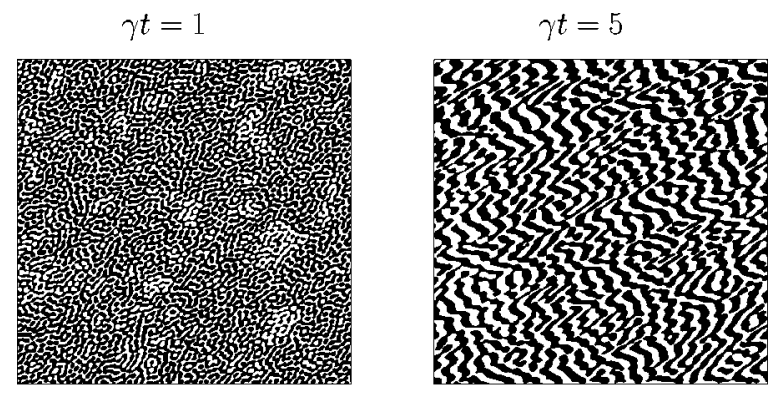

$\gamma t=11$
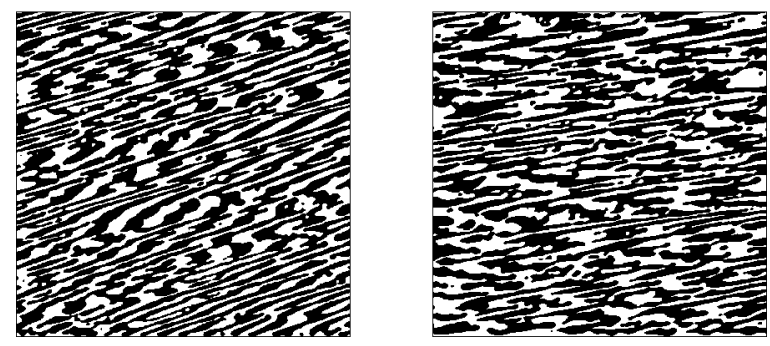

FIG. 1. Configurations of a portion of $512 \times 512$ sites of the whole lattice are shown at different values of the strain $\gamma t$ at $T=0$. The $x$ axis is in the horizontal direction.

and analogously for the other directions. Of experimental interest are also the rheological indicators, among which the excess viscosity defined as $[6,22]$

$$
\Delta \eta=-\frac{1}{\gamma} \int \frac{d \vec{k}}{(2 \pi)^{d}} k_{x} k_{y} C(\vec{k}, t) .
$$

We have simulated Eq. (2) in $d=2$ and 3 by a first-order Euler discretization scheme. Periodic boundary conditions have been implemented in the $x$ and $z$ directions (for the $d=3$ case); Lees-Edwards boundary conditions [23] were used in the $y$ direction. These boundary conditions, originally developed for molecular dynamics simulation of fluids in shear, require the identification of a point at $(x, 0, z)$ with one located at $(x+\gamma L \Delta t, L, z)$, where $L$ is the size of the lattice (the same in all the directions) and $\Delta t$ is the time discretization interval. The system was initialized in a high temperature disordered state, and the evolution was studied with $a$ $<0$. Simulations were run using lattices of size $L=1024$ in $d=2$ and $L=256$ in $d=3$ with $\Delta x=0.5,1$. The width of interfaces is given by $\sqrt{2 \kappa}$ [24]. We do not observe significant differences between these two choices of $\Delta x$. The results here shown were obtained with $\gamma=0.0488, \Delta x=1$, $\Delta t=0.01$, and $\langle\varphi\rangle=0$. The two-dimensional system was studied considering temperatures in the range $0 \leqslant T \leqslant 5$, while only the $T=0$ case was studied in $d=3$.

\section{NUMERICAL SIMULATIONS: $d=2$}

$$
\text { A. } T=0
$$

In this section we discuss the results of the numerical simulations of Eq. (2) without the thermal noise $\eta$. A sequence of configurations of the order parameter are shown in Fig. 1 at different values of the strain $\gamma t$. After a typical early stage, when domains are formed from the mixed initial state, a bicontinuous structure is observed. The distortions pro-

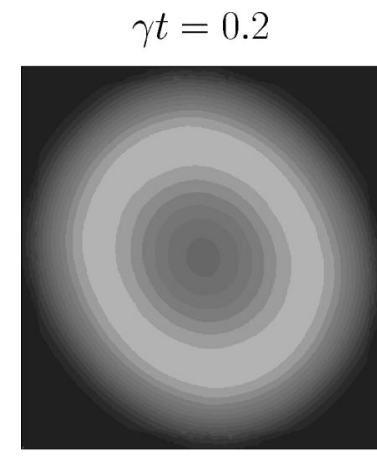

$\gamma t=11$

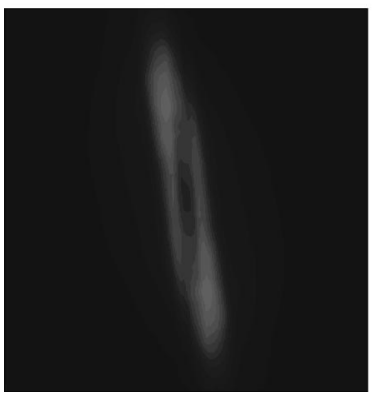

FIG. 2. The structure factor is shown at different values of the shear strain $\gamma t$ at $T=0$. The axes $k_{x}$ and $k_{y}$ are in the horizontal and vertical directions, respectively.

duced by the flow appear evident from $\gamma t \simeq 1$ onwards. At $\gamma t=11$, striped domains aligned with a tilt angle $\theta$ with respect to the flow are observed. As time elapses $\theta$ decreases, and the structures align with the flow. In the meanwhile nonuniformities are formed in the system: Regions with domains of different thickness can be clearly observed. Small bubbles are also present, originating from the fragmentation of strained domains.

A more accurate analysis of the spatial properties can be achieved by means of the structure factor, shown in Fig. 2. Initially $C(\vec{k}, t)$ exhibits an almost circular shape, corresponding to the early stage. Then the presence of shear deforms $C(\vec{k}, t)$ into an ellipse, and modifies the profile of the edge until, for $\gamma t \geqslant 1$, four peaks can be clearly observed. The presence of a peak in the structure factor is generally interpreted as the signature of a characteristic length in the fluid. Here, due to the anisotropy, for each peak one associates one length with each spatial dimension. Since the peaks are related by the symmetry $\vec{k} \rightarrow-\vec{k}$, one concludes that there are two distinct characteristic lengths for each space direction. This corresponds to the observation of thinner and thicker domains in Fig. 1. The relative height of the peaks in one of the two specular foils in which $C(\vec{k}, t)$ is separated, starting from $\gamma t \simeq 4$, is better seen in Fig. 3. For a better view of $C(\vec{k}, t)$, we have enlarged the scales on the $k_{x}$ and $k_{y}$ axes differently. Here one observes that the two peaks dominate alternately at the times $\gamma t=11$ and 20. In a situation like that at $\gamma t=11$, the peak with the larger $k_{y}$ dominates, indicating that the stretched thin domains are more abundant. When the strain is further increased their rupture makes the contribution of the thick regions more important; this causes the other peak of $C(\vec{k}, t)$ to dominate. This mechanism is very reminiscent of what happens in the large- $N$ model, where the 


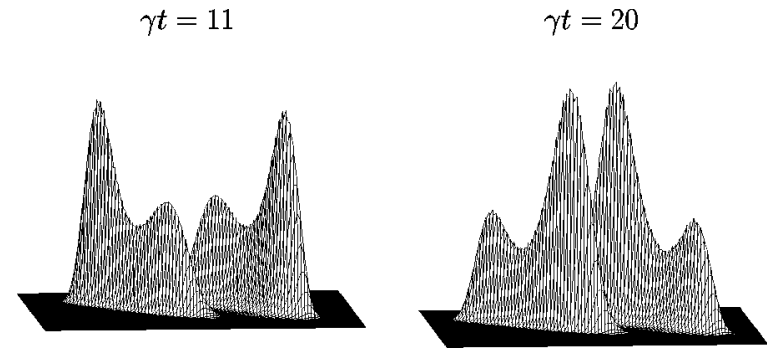

FIG. 3. The same structure factor as in Fig. 2 is shown in a three-dimensional plot at $\gamma t=11$ and $\gamma t=20$ in order to better illustrate the different relative heights of the peaks at different times. The axes $k_{x}$ and $k_{y}$ are in the horizontal and vertical directions, respectively.

recurrent prevalence of the peaks is shown to reproduce periodically in time up to much longer times then those reachable in the simulations here presented.

The mechanism of stretching and breakup which characterizes the domain evolution produces an oscillatory pattern in the typical size of ordered regions $R_{x}$ and $R_{y}$. This is shown in the first picture of Fig. 4 [25]. $R_{y}$ reaches a local maximum when $C(\vec{k}, t)$ is of the form of Fig. 3 at $\gamma t=20$. As already observed, thick domains are more abundant at this time, as one can see in Fig. 1 at $\gamma t=20$. In the large- $N$ model this oscillatory behavior is periodic in the logarithm of time, and is superimposed on an average power-law increase of the lengths $R_{x}$ and $R_{y}$. Here CPU limitations prevent a clear check of both these predictions. This behavior reflects itself on most physical observables, among which the excess viscosity. This is shown in the first picture of the panel of Fig. 5 where $\Delta \eta$ is plotted against the shear strain $\gamma t$. Starting from zero in correspondence with the isotropic initial condi-
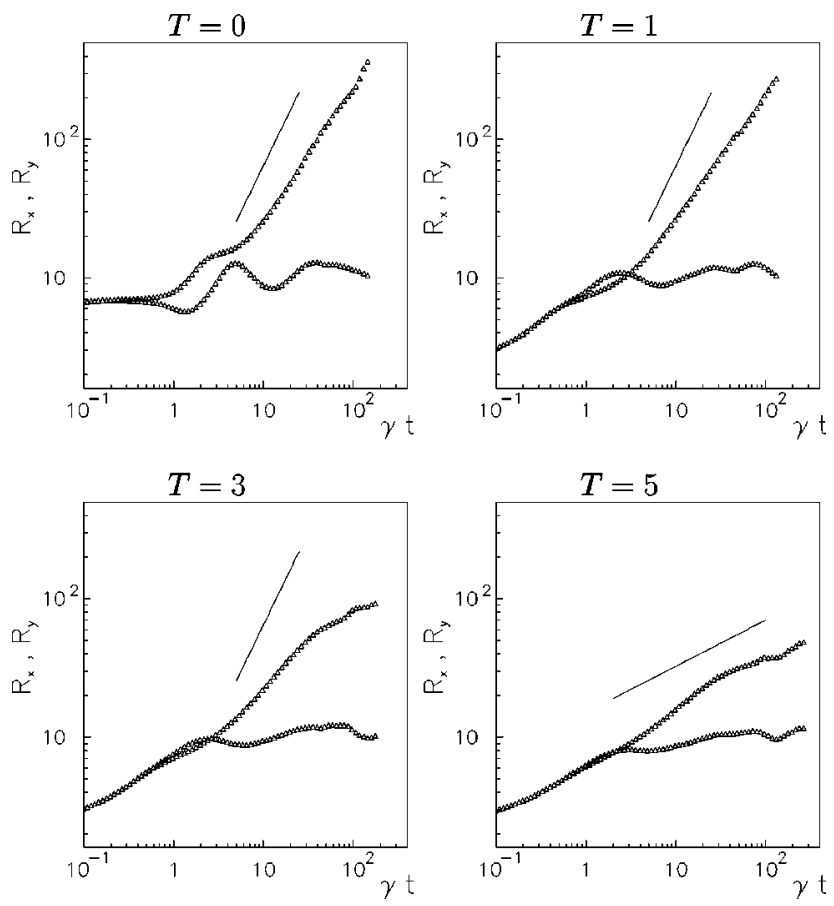

FIG. 4. The average size of domains in the flow (upper curve) and in the shear direction (lower curve) are plotted as functions of the shear strain $\gamma t$ at different temperatures. The straight lines have slopes $4 / 3$ at $T=0,1$, and 3 , and $1 / 3$ at $T=5$.
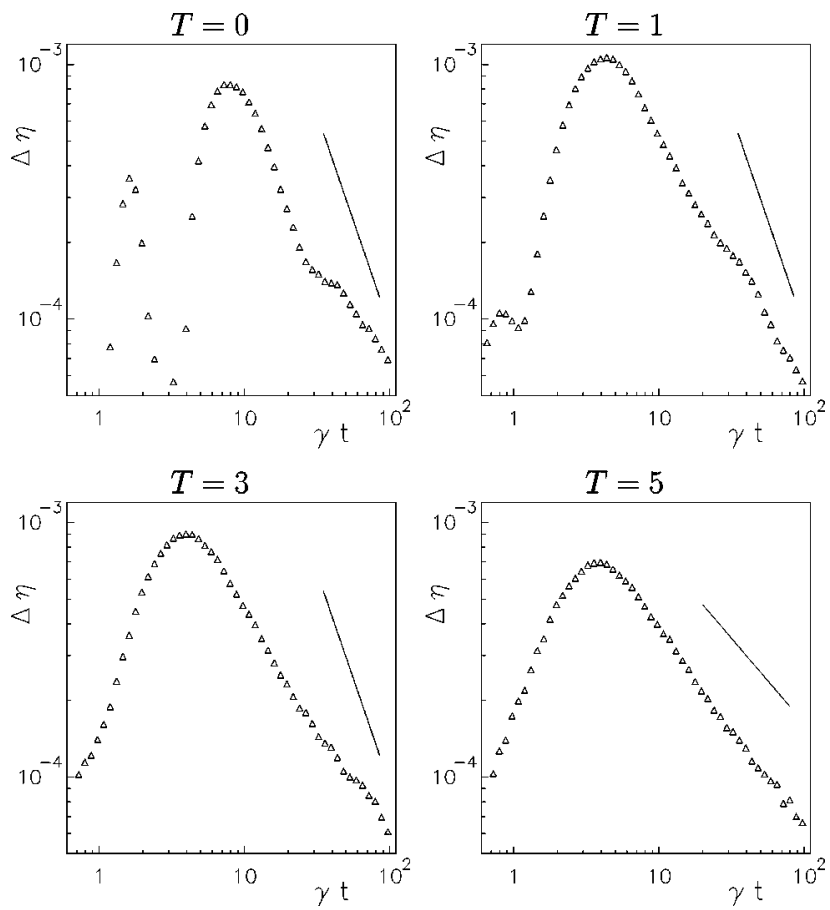

FIG. 5. The excess viscosity is plotted as a function of the shear strain $\gamma t$ at different temperatures. The straight lines have slopes $-5 / 3$ at $T=0,1$, and 3 , and $-2 / 3$ at $T=5$.

tion, $\Delta \eta$ shows a net increase up to a global maximum at $\gamma t \simeq 8$. This is because the stretching of the domains caused by the shear requires work against surface tension. This increase, however, is not monotonic, and $\Delta \eta$ shows an oscillating pattern with a first pronounced local maximum at $\gamma t \simeq 1.5$. For larger times $\Delta \eta$ decreases due to the ordering process in the fluid. Figure 5 suggests that oscillations decorate the behavior of the excess viscosity even in this regime. Another oscillation is completed at $\gamma t \simeq 40$. This is further supported by the analogous feature in the large- $N$ model. We mention that a double overshoot of $\Delta \eta$ is also observed in experiments [18].

\section{B. Role of temperature fluctuations}

We have performed numerical simulations of Eq. (2) for several temperatures in the range $0-5$. Generally speaking, we observe a qualitative behavior similar to that in the zero temperature case. In particular, from the analysis of the field configurations presented in Fig. 6 for $T=5$, one again observes an anisotropic deformation of the bicontinuous pattern emerging after the linear regime and the inhomogeneities in the domain thickness. Thermal fluctuations are responsible for the increased roughness of the interfaces with respect to $T=0$ and for the thermal excitations inside the ordered domains, similar to the case without shear.

The structure factor, shown in Fig. 7, starting from $\gamma t=4$, exhibits four peaks whose relative heights prevail recurrently. However, the difference of the heights of the peaks on the same foil is less than in the case $T=0$. At $\gamma t=1$, instead, one has only two broad maxima along the diagonal $k_{x} \simeq k_{y}$ that is split into four peaks only at later times. The same behavior is also observed at $T=0$, but for earlier times. 
$\gamma t=3$

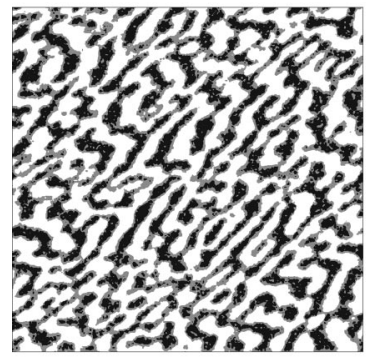

$\gamma t=13$

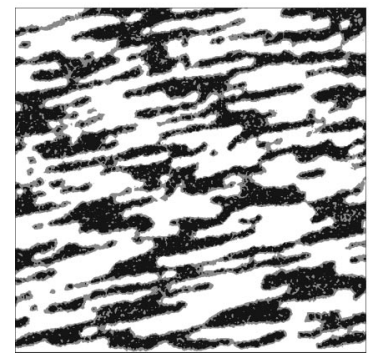

$\gamma t=7$

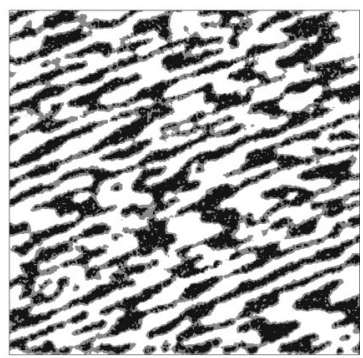

$\gamma t=17$

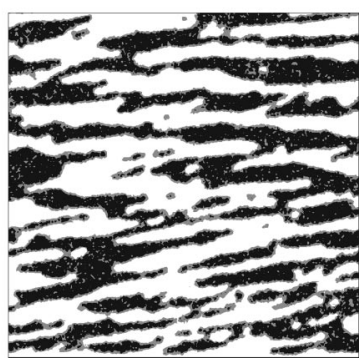

FIG. 6. Configurations of a portion of $256 \times 256$ sites of the whole lattice are shown at different values of the strain $\gamma t$ at $T$ $=5$. The $x$ axis is in the horizontal direction. Grey scaling from black to white corresponds to values of $\varphi$ from -1 to 1 .

This suggests that thermal fluctuations slow the evolution of $C(\vec{k}, t)$.

Quantitative differences from the case $T=0$, possibly related to the temperature-induced slower evolution, are exhibited by the characteristic sizes of the domains in the $x$ and $y$ directions. These quantities are compared for several temperatures in Fig. 4. On the basis of renormalization group [19] or scaling arguments [16], $R_{y}$ and $R_{x}$ are expected to
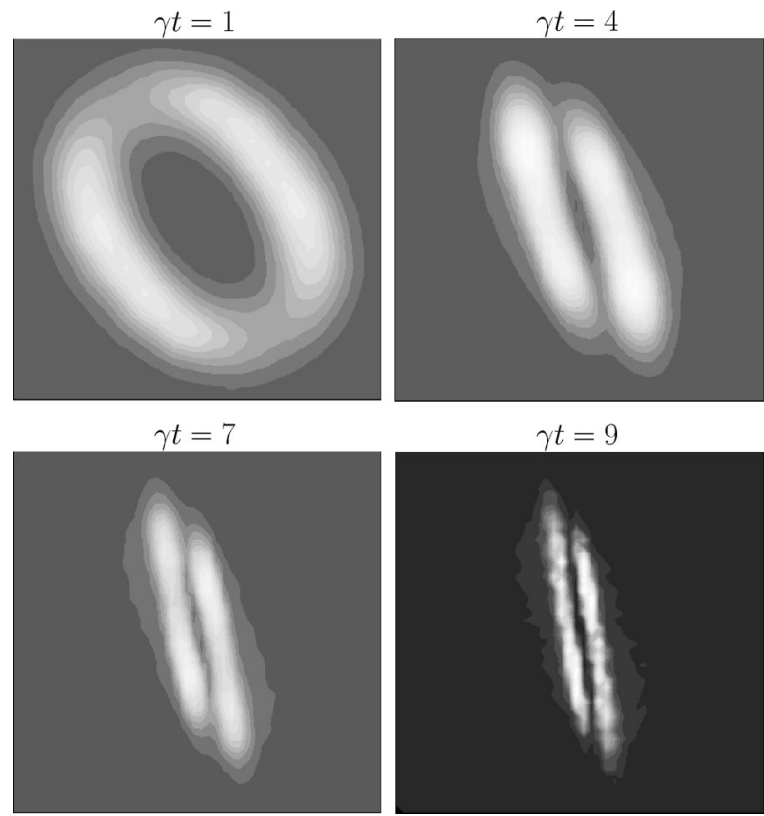

FIG. 7. The structure factor is shown at different values of the shear strain $\gamma t$ at $T=5$. The axes $k_{x}$ and $k_{y}$ are in the horizontal and vertical directions, respectively. obey an asymptotic power-law growth $t^{\alpha}$ with $\alpha_{y}=1 / 3$ and $\alpha_{x}=\alpha_{y}+1$. The exponent $\alpha_{y}$ is the same as the exponent without shear, and is therefore presumably caused by the evaporation-condensation mechanism responsible for growth in the absence of flow. On the other hand, the growth law in the flow direction is induced by the shear. An accurate determination of these exponents is not possible on the basis of our numerical simulations. Finite-size effects become relevant in the phase separation process quite quickly. Moreover, the possible power-law behaviors are decorated by oscillations, and it would be necessary to access much longer times in order to average over this periodic contribution. Due to finite-size effects this, in turn, would require even larger lattices, which is too numerically demanding for standard workstations. Despite these limitations, by looking at Fig. 4 one can still make some qualitative observation. At $T=0$ one observes for $\gamma t>30$ a regime roughly consistent with $\alpha_{x}-\alpha_{y}=1$, even if $\alpha_{x}$ is smaller then the expected value $4 / 3$ and $\alpha_{y}$ is not even well defined because of large oscillations. By raising the temperature one sees that this regime is gradually changed, and the effective exponent $\alpha_{x}$ is lowered until, at $T=5$, it becomes comparable to the value $1 / 3$ observed without shear. If one believes that the asymptotic exponents are not changed by the strength of thermal fluctuations, as suggested by the renormalization group argument [19] mentioned above, and by the analogy with the case $\gamma=0$, then the role of the temperature is that of producing a preasymptotic behavior characterized by a slower growth in the direction of the flow.

In binary mixtures without shear the temperature is known to help the segregation process by increasing the value of the amplitude in the expression of $R(t) \sim A t^{1 / 3}$ $[2,21]$. Here, as an effect of the increased temperature, the contribution to the phase separation of the usual evaporationcondensation mechanism, associated with the power exponent $1 / 3$, becomes more relevant than the effects of the shear induced mechanism. This results in the observed preasymptotic behavior $R_{x}=A t^{1 / 3}$. At later times, even if not observed in our simulations, we expect a growth with a larger exponent close to $4 / 3$.

The above trend is reflected in the behavior of the excess viscosity shown in Fig. 5. If scaling is obeyed asymptotically, so that $C(\vec{k}, t) \sim R_{x} R_{y} f\left(k_{x} R_{x}, k_{y} R_{y}\right)$, then, from Eq. (6), one would expect $\Delta \eta$ to scale as the inverse domain volume, namely, $\Delta \eta \sim R_{x}^{-1} R_{y}^{-1}$. Hence, given the above discussed behavior of $R_{x}$ and $R_{y}$ as the temperature is changed, one expects $\Delta \eta$ to gradually cross over from a power law $t^{-5 / 3}$ at low temperatures to $t^{-2 / 3}$ at higher temperatures, which is roughly consistent with our data.

Finally we make some observations about the influence of the temperature on the oscillating behavior. The role of the oscillations is progressively suppressed increasing $T$. This is observed in Fig. 5. The most apparent effect is the disapparence for $T>1$ of the initial double overshoot of $\Delta \eta$. A gradual suppression of oscillations due to thermal fluctuations is also observed in the plot of $R_{x}$ and $R_{y}$ (Fig. 4). This is consistent with the above discussion of the effects of raising the temperature.

\section{NUMERICAL SIMULATIONS: $d=3$}

In this section we present the results of numerical simulations of the zero temperature quench process in a three- 


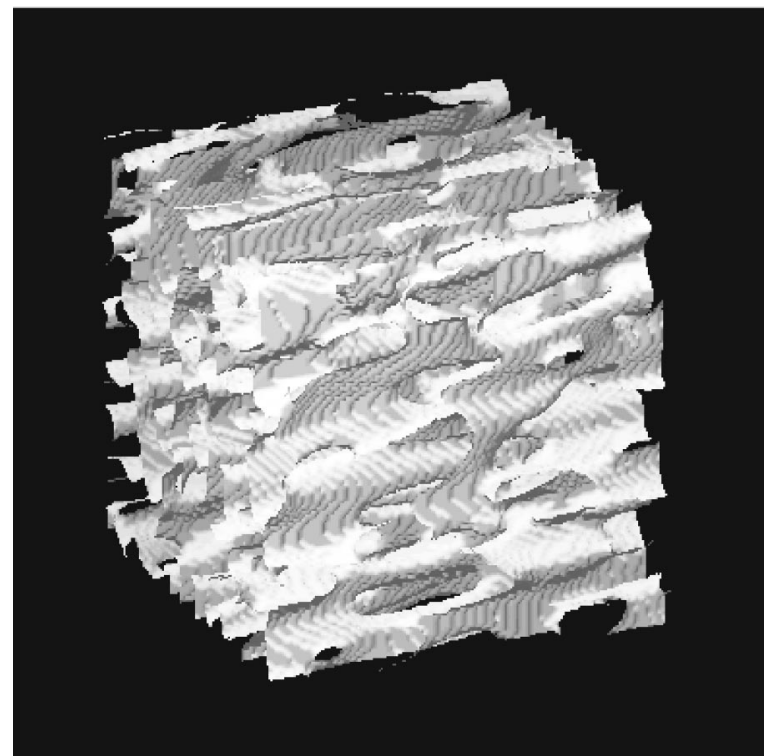

FIG. 8. The interfaces between domains of different compositions of a $128^{3}$ section of the simulated system are shown at $T$ $=0$. The $x$ axis points from left to right, the $y$ axis points into the foil, and the $z$ axis points from bottom to top.

dimensional system. The typical structures produced in the coarsening process are shown in Fig. 8. Domains appear with an interconnected cylindrical shape aligned with a tilt angle $\theta$ that shrinks in time with the flow direction. Contour plots of the structure factor along the coordinate planes are presented in Fig. 9. On the plane $k_{x}=0$ the shear term drops out from Eq. (2), as can be easily checked by transforming Eq. (2) into reciprocal space. Then shear is ineffective along this direction, and the structure factor displays the usual circular form as for a static fluid. In the plane $k_{y}=0, C(\vec{k}, t)$ has an elliptical form with axes along $k_{x}$ and $k_{y}$. The white spots reveal that four peaks with the same height are present. In the plane $k_{z}$, a behavior reminiscent of the two-dimensional case is observed. These results can be compared, and better describe the experimental measures of the structure factor of Ref. [26]. As in $d=2$, the periodic modulation of the relative heights of the different peaks produces an oscillatory behavior of most observables, as shown in Fig. 10 for the characteristic sizes $R_{x}, R_{y}$, and $R_{z}$, and in Fig. 11 for the excess viscosity $\Delta \eta$. While the oscillations in the $x$ and $y$ directions are out of phase, due to the recurrent prevalence of thin and thick domains described in the two-dimensional system, it is interesting to note that $R_{z}$ has a different phase with respect

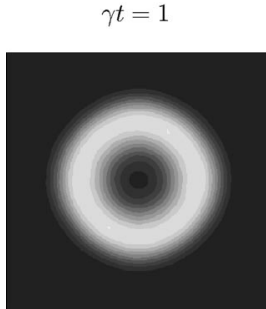

$k_{x}=0$ $\gamma t=3$

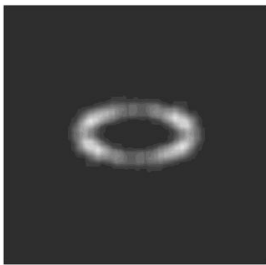

$k_{y}=0$ $\gamma t=1$

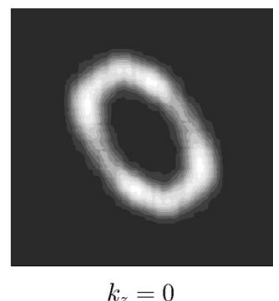

FIG. 9. The sections $k_{x}=0, k_{y}=0$, and $k_{z}=0$ of the structure factor are shown at $\gamma t=1,3$, and 1 , respectively, and $T=0$.

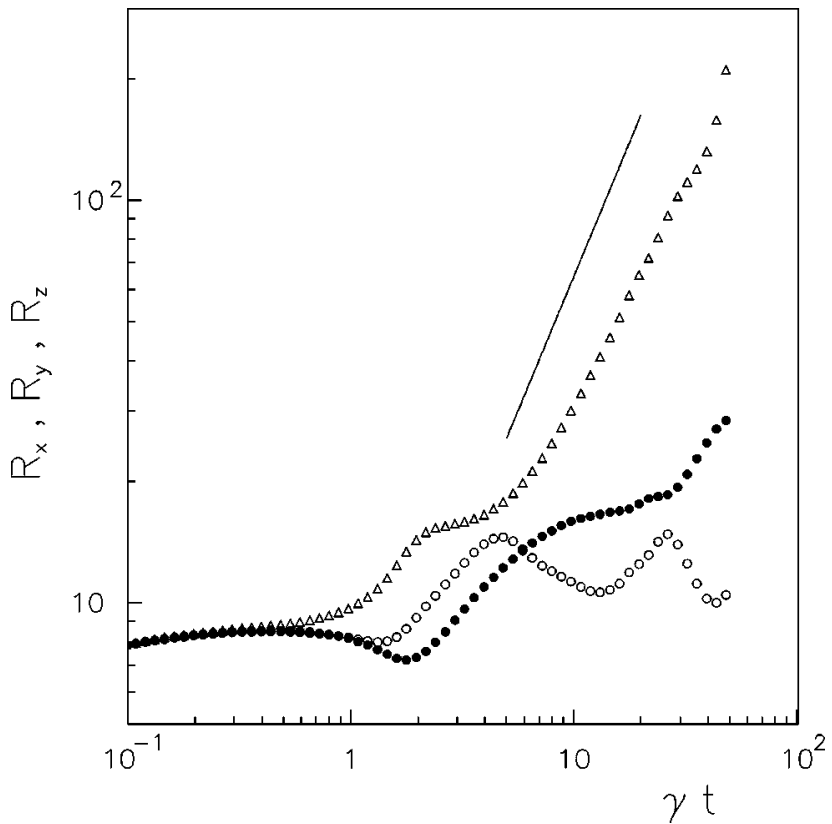

FIG. 10. Evolution of the average domain size in the $x(\triangle)$, $y(\bigcirc)$, and $z(\bigcirc)$ directions at $T=0$. The straight line has a slope $4 / 3$.

to $R_{y}$, indicating an even richer behavior. These results provide at least an indication of a power-law increase of $R_{x}$ and $R_{z}$, apart from the oscillations, with an exponent larger than 1.2 for $R_{x}$. In the $y$ direction, however, there is no evidence of growth.

\section{CONCLUSIONS}

In this paper we have considered the phase separation process of a fluid binary mixture in the presence of an ap-

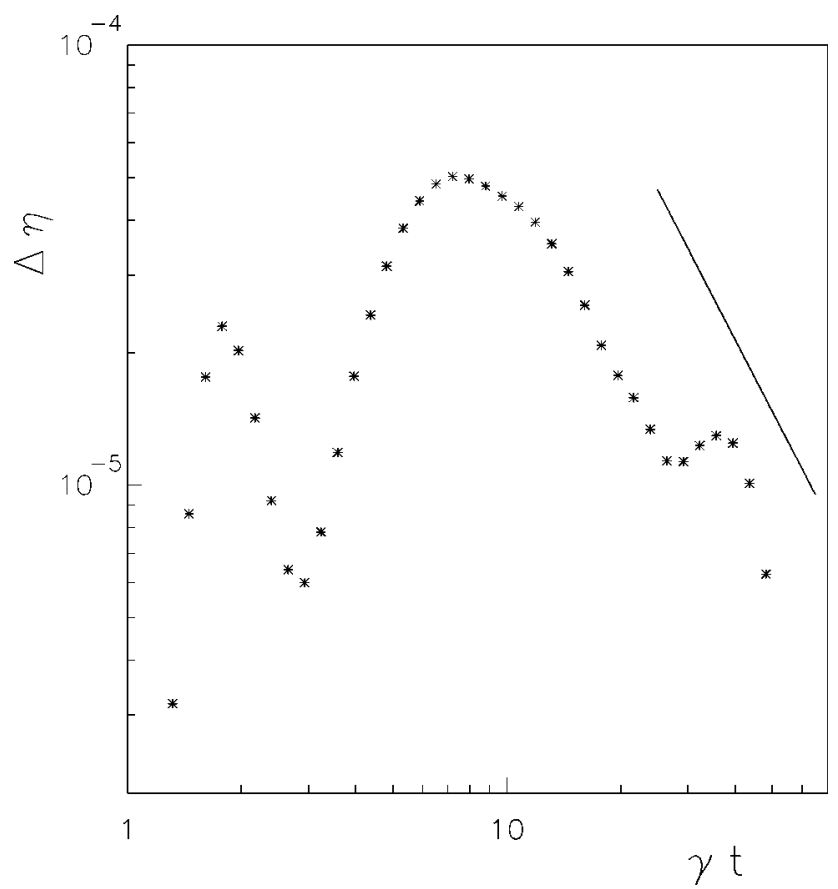

FIG. 11. The excess viscosity is plotted as function of the shear strain $\gamma t$ at $T=0$. The straight line has a slope $-5 / 3$. 
plied plane shear flow. The binary mixture is described by a continuum free-energy functional, and we have numerically studied the corresponding time-dependent Ginzburg-Landau equation with a convective term introduced by the flow. Results have been presented for two-dimensional systems quenched to different final temperatures, and for threedimensional fluids quenched to $T=0$. Simulations were carried out on lattices larger than those usually considered in phase separation studies, in order to obtain the highest possible resolution for the structure factor.

In real binary fluids the existence of a four-peaked structure factor was reported [17], together with a double overshoot of the excess viscosity. To our knowledge, however, a coherent picture indicating a possible connection between these apparently different phenomena with a clear physical interpretation of their origin was not available. The results of this paper strongly suggest that the interplay between the four peaks of the structure factor, with their recurrent prevalence, gives rise to an oscillatory phenomenon which reflects itself on the main observables. On these bases we conjecture that the double overshoot observed in experiment can be interpreted as the first part of an oscillatory pattern superimposed on the global trend of the excess viscosity, and that other local maxima of this quantity could be observed in an experiment on longer time scales.

Our simulations suggest that the physical origin of the oscillating behavior is related to the presence of two kind of domains, characterized by different sizes. The kinetics proceeds by stretching the thicker domains and breaking the thinner ones, so that a cyclical prevalence of one of the two types occurs. This is reflected on the properties of the structure factor and other quantities. These results agree at a semiquantitative level with the pattern observed in the large- $N$ approximation [1], indicating that the global picture put forward by the $N=\infty$ theory is reliable, despite the different physical character of the large- $N$ system without stable topological defects. This prediction can be used to infer that the observed oscillations are periodic in the logarithm of time, and that the phase separation proceeds via dynamical scaling, so that the characteristic sizes of the domains grow according to power laws whose expected exponents were computed in Refs. [16,19]. However, these conjectures could not be clearly addressed by our simulations with the available CPU resources, because a much larger system should be considered at much longer times.

\section{ACKNOWLEDGMENTS}

F.C. is grateful to M. Cirillo, R. Del Sole, and M. Palummo for hospitality at the University of Rome. F.C. and G.G. acknowledge support by the TMR network Contract No. ERBFM-RXCT980183 and by PRA-HOP 1999 INFM.
[1] F. Corberi, G. Gonnella, and A. Lamura, Phys. Rev. Lett. 81, 3852 (1998).

[2] K. Binder in Phase Transitions in Materials, Materials Science and Technology Vol. 5, edited by R. W. Cahn, P. Haasen, and E. J. Kramer (VCH, Weinheim, 1990); H. Furukawa, Adv. Phys. 34, 703 (1985); J. D. Gunton, M. San Miguel, and P. S. Sahni, in Phase Transitions and Critical Phenomena, edited by C. Domb and J. L. Lebowitz (Academic, New York, 1983), Vol. 8; A. J. Bray, Adv. Phys. 43, 357 (1994).

[3] I. M. Lifshitz and V. V. Slyozov, J. Phys. Chem. Solids 19, 35 (1961).

[4] A. Coniglio, P. Ruggiero, and M. Zannetti, Phys. Rev. E 50, 1046 (1994); F. de Pasquale, G. F. Mazenko, P. Tartaglia, and M. Zannetti, Phys. Rev. B 37, 296 (1988); S. C. Glotzer and A. Coniglio, Phys. Rev. E 50, 4241 (1994); U. Marini Bettolo Marconi and F. Corberi, Europhys. Lett. 30, 349 (1995).

[5] See, e.g., R. G. Larson, The Structure and Rheology of Complex Fluids (Oxford University Press, New York, 1999).

[6] For a review, see A. Onuki, J. Phys.: Condens. Matter 9, 6119 (1997).

[7] J. Läuger, C. Laubner, and W. Gronski, Phys. Rev. Lett. 75, 3576 (1995).

[8] P. Padilla and S. Toxvaerd, J. Chem. Phys. 106, 2342 (1997).

[9] T. Hashimoto, K. Matsuzaka, E. Moses, and A. Onuki, Phys. Rev. Lett. 74, 126 (1994).

[10] R. Yamamoto and X. C. Zeng, Phys. Rev. E 59, 3223 (1999).

[11] Z. Shou and A. Chakrabarti, Phys. Rev. E 61, R2200 (2000).

[12] H. Liu and A. Chakrabarti, J. Chem. Phys. 112, 10582 (2000).

[13] A. H. Krall, J. V. Sengers, and K. Hamano, Phys. Rev. Lett. 69, 1963 (1992).
[14] D. H. Rothman, Europhys. Lett. 14, 337 (1991).

[15] T. Ohta, H. Nozaki, and M. Doi, Phys. Lett. A 145, 304 (1990); J. Chem. Phys. 93, 2664 (1990).

[16] N. P. Rapapa and A. J. Bray, Phys. Rev. Lett. 83, 3856 (1999).

[17] K. Migler, C. Liu, and D. J. Pine, Macromolecules 29, 1422 (1996).

[18] Z. Laufer, H. L. Jalink, and A. J. Staverman, J. Poly. Sci. Polym. Chem. Ed. 11, 3005 (1973); S. Mani, M. F. Malone, H. H. Winter, J. L. Halary, and L. Monnerie, Macromolecules 24, 5451 (1991); S. Mani, M. F. Malone, and H. H. Winter, ibid. 25, 5671 (1992).

[19] F. Corberi, G. Gonnella, and A. Lamura, Phys. Rev. Lett. 83, 4057 (1999).

[20] A recent review on the influence of hydrodynamic effects on scaling properties was presented by J. M. Yeomans, Annu. Rev. Comput. Phys. VII (2000). For simulations with hydrodynamic models, see M. E. Cates, V. M. Kendon, P. Bladon, and J. C. Desplat, Faraday Discuss. 112, 1 (1999); A. J. Wagner and J. M. Yeomans, Phys. Rev. E 59, 4366 (1999); A. Lamura and G. Gonnella, e-print cond-mat/0005518.

[21] T. M. Rogers, K. R. Elder, and R. C. Desai, Phys. Rev. B 37, 9638 (1988).

[22] A. Onuki, Phys. Rev. A 35, 5149 (1987).

[23] A. W. Lees and S. F. Edwards, J. Phys. C 5, 1921 (1972).

[24] J. S. Rowlinson and B. Widom, Molecular Theory of Capillarity (Clarendon Press, Oxford, 1982).

[25] The characteristic lengths $R(t)$ are measured in units of $\sqrt{\kappa / a}$, and time in terms of $\gamma a^{2} / \kappa$.

[26] C. K. Chan, F. Perrot, and D. Beysens, Phys. Rev. A 43, 1826 (1991). 